\title{
Sample Audit of Cards from a University Library Catalog
}

This paper reviews results of studying a sample of cards (an audit) from a catalog, indicates uses, and suggests perspectives for other audit operations.

\begin{abstract}
A Ny LaRge Card Catalog will contain a certain number of errors, conflicts, mutilated cards, and examples of obsolete and misleading cataloging practices designed for the catalog when it was much smaller. The perfect catalog does not exist, and the nearly perfect catalog is very often counterbalanced by hoards of "problem" catalog cards stashed away in the catalog department. Although the state of maintenance of their catalogs is a universal concern of catalog librarians, this concern is not always based on reliable data on the actual condition of the catalog. One means of acquiring this information is to audit a sample of cards from the catalog for errors and inadequacies. Information provided by such an audit can be used for setting task priorities and manpower requirements for retroactive maintenance and, when performed periodically, for measuring the effectiveness of programs of current maintenance.
\end{abstract}

The author-title section of the main public catalog at the University of Colorado Libraries has been audited, primar-

At the time this paper was written, $\mathrm{Mr}$. Hewitt was head, Catalog Maintenance Department, University of Colorado Libraries, Boulder. He is presently coordinator, Colorado Academic Libraries Book Processing Center (CALBPC), Boulder. ily for the purpose of setting priorities for projects of rehabilitation. Based on the information provided by the audit, the following projects were given top priority: (1) Updating of cross-reference structure of the catalog; (2) systematic search and replacement of mutilated cards; and (3) replacement of the more misleading short cards, especially added entries in the form of see references. The audit allows us to concentrate our limited resources for maintenance on the most pressing projects, without the nagging suspicion that there are other projects of more critical immediacy. It is noteworthy that the weakness of the cross-reference structure of the catalog was not suspected before the audit. The sample audit, though substantially lacking in several ways, has proven well worth the time and trouble spent in conducting it.

A search of the literature for reports of similar audits of university library catalogs produced only meager results. Two possible explanations for this lack of literature come to mind: (1) The sample catalog audit is a neglected or undiscovered tool in research libraries; or (2) the results of audits are considered too localized in interest-or too embarrassing-to be reported in the literature. In either case, the unfortunate result is that the research library performing an audit does so in a vacuum. Al- 
though the primary usefulness of an audit is certainly not to compare catalogs with other libraries, auditing without comparative data can be an acute disadvantage when reporting audit results to the staff. Without comparative statistics from other libraries the staff may compare error and card deficiency rates with the only yardstick availableabsolute perfection. The result may be that the catalog department may plunge, or be pushed, into a costly and unnecessary project of editing and refiling the catalog.

This report attempts to break the vacuum's seal, regardless of its cause. This paper will demonstrate how audit information has been useful in one university library, and it may lend perspective to audits being performed elsewhere.

The divided catalog at the University of Colorado's Norlin Library consists of approximately $1,000,000$ cards in the author-title section and 600,000 in the subject section. Only the author-title section of the catalog was audited. (The audit required forty-four hours of professional time, not including the time required to write a final report and recommendations.) The sample totaled 2,500 cards, consisting of the first 100 cards in a random sample of twenty-five trays. The confidence level for the sample is 95 percent, with precision intervals varying according to the proportions of each attribute tabulated. Approximate precision levels were determined by the use of a sampling table, and all were considered adequate for the purposes of the study. ${ }^{1}$ For example, the precision level for the filing error rate is approximately .005 . With a filing error rate of 1.1 percent in the sample, we can be assured that in ninety-five times out of a hundred the actual filing error rate in the catalog will fall between .6 percent and 1.6 percent.

The composition of the sample by type of entry was as follows: Main en- tries, including primary entries for analytical sets-42.8 percent; title added entries-24.2 percent; added author, editor, and translator entries-13 percent; added series entries-7.4 percent; continuation cards ( second cards to a given entry)-4.4 percent; references-5.3 percent; information cards-1 percent; and guide cards-1.1 percent.

The rate of filing error in the sample was 1.1 percent. Errors resulting from a lack of knowledge of the filing rules made up 52 percent of the errors in the sample. The remaining 48 percent were mechanical or simple alphabetical errors. The filing of an added entry in a separate sequence after the same heading used as a main entry was the most frequent error involving violation of a filing rule. Since this reflects an earlier filing practice, it may be assumed that a number of these had not been refiled when the library's present rule was adopted. Other frequent rule errors were subfiling by main entry rather than title, mistaking a subdivision of a corporate body for a title, and the filing of complete works in foreign languages alphabetically rather than before the sequence for the author's single works. In most cases errors involving violation of a filing rule did not result in loss of access through the misfiled entry, since the card was usually somewhere within the sequence for the heading. Alphabetical errors tended to misplace cards much more drastically.

Initially, some staff members were alarmed at our filing error rate. After all, a rate of 1.1 percent means approximately 11,600 filing errors in the authortitle catalog alone. The staff member who found some mystical scale to convert this statistic into a patron inconvenience factor was truly staggered. Fortunately, figures were available from the 1953 audit of the official catalog at the Library of Congress, which reported a filing error rate "in excess of 5 percent." 2 This served to temper consider- 
ably the reaction to our own error rate.

Of the cross-references in the sample 10.14 percent were blind, resulting in an estimate of 5,600 blind references in the catalog. The weakness of the crossreference structure in the author-title section of the catalog was completely unexpected, and this statistic instigated a search for an offending procedure. The main cause has been identified as a gap in the cancellation process. In cancelling the last card under a heading, it had not been the practice to check systematically the authority file to see if references were made to that entry. The audit demonstrated that such a lapse, over a period of time, can have a considerable effect on the catalog. Several of the blind references had not been transferred into the subject section when the catalog was divided.

Of the cards in the sample 1.48 percent were judged to be mutilated. Since mutilation was strictly defined to include only cards with the call number or other essential information torn off or completely obscured by dirt and wear, this statistic may only be used to estimate the number of cards needing immediate replacement. There were a number of cards in the sample in various stages of deterioration which were not counted as mutilated. For that reason the study was of little help in estimating manpower requirements for retyping over the next several years. The lack of more intensive analysis of card deterioration is a major deficiency of the study, and in future audits estimates will be made of the length of usefulness of each card in the sample.

Other card deficiencies recorded were obsolete cards (page analytics for periodicals, analytics to pamphlet collection) -.8 per cent; short cards for added entries-7.3 percent of added entries; abbreviated cataloging (adding new editions as added copies )-1.1 percent.

Several card characteristics were recorded for purposes other than deter- mining the state of maintenance of the catalog. The incidence of tracings on the verso rather than the face of the main entry was recorded for information useful in considering a proposal to reproduce our present catalog in book form by photographic means. Thirtynine percent of the main entries had complete or partial tracings on the verso of the card, pointing out the considerable cost of retaining a visible record of tracings in a book catalog. Two statistics that will be useful in considering the question of reclassification are that 35 percent of the cards in the sample for titles classed in Dewey were on LC printed cards with usable class numbers, and that 90 percent of card deficiencies (not including filing errors) were on Dewey cards.

As stated earlier, the primary use of the audit has been to set task priorities for maintenance projects. However, a fairly reliable estimate of the rate of filing error in the catalog may well turn out to be the most valuable statistic provided by the audit. Like many libraries, we are presently seeking relief from the considerable manpower drain of complete filing revision (student filers, clerical revisers). Several alternatives to complete revision are being considered. Among these are nonrevision, sample revision, and partial or selective revision. If either of these alternatives is adopted, our present filing error rate will be a base figure by which to measure the effect of the new method on the filing in the catalog.

The auditing technique becomes more advanced in its application to the problem of filing error, from which a system of error control based on the periodic sample audit and an established level of tolerable filing error may evolve. The difficult problem here will not be perfecting the auditing process, but rather establishing a realistic level of acceptable filing error. It is here that comparative statistics would be most 
useful in making this decision much less arbitrary.

\section{REFERENCES}

1. Gene Brown and Lawrence L. Vance, Sampling Tables for Estimating Error Rates or
Other Proportions (Berkeley: Institute of Business and Economic Research, University of California, 1961). Table II, p.52.

2. Cited in Andrew D. Osborn and Susan M. Haskins, "Catalog Maintenance," Library Trends 2:280 (Oct. 1953). 